\title{
Psychosocial Influences on Pregnancy and Childbirth Behaviors in Northwestern Nigeria: A Cross-Sectional Analysis
}

\section{Emily White Johansson ( $\nabla$ ejohansson@tulane.edu )}

Tulane University School of Public Health and Tropical Medicine https://orcid.org/0000-0001-5162-8277

\section{Udochisom Anaba}

Tulane University School of Public Health and Tropical Medicine / Breakthrough Research Nigeria

\section{Dele Abegunde}

Population Council / Breakthrough Research Nigeria

Mathew Okoh

Johns Hopkins University Center for Communication Programs / Breakthrough Action Nigeria

Shittu Abdu-Aguye

Johns Hopkins University Center for Communication Programs / Breakthrough Action Nigeria

Paul C Hewett

Population Council / Breakthrough Research Nigeria

Paul L Hutchinson

Tulane University School of Public Health and Tropical Medicine / Breakthrough Research Nigeria

\section{Research article}

Keywords: Nigeria, pregnancy, ideation, psychosocial, survey

Posted Date: December 10th, 2020

DOI: https://doi.org/10.21203/rs.3.rs-124239/v1

License: (c) (i) This work is licensed under a Creative Commons Attribution 4.0 International License. Read Full License 


\section{Abstract}

\section{Background}

Antenatal care (ANC) and facility delivery are essential maternal health services, but uptake remains low in northwestern Nigeria. To increase service use, social and behavior change (SBC) programs target psychosocial influences across cognitive, emotional and social domains including knowledge, beliefs, selfefficacy, and social norms. Yet there remains limited research that measures and quantitatively examines the role of psychosocial influences on pregnancy and childbirth behaviors in Nigeria or elsewhere.

\section{Methods}

A cross-sectional population-based survey of randomly sampled women with a child under two years was conducted in Kebbi, Sokoto and Zamfara states of northwestern Nigeria in September 2019. Women were asked about maternal health behaviors during their last pregnancy. New psychosocial metrics were developed using the Ideation Model of Strategic Communication and Behavior Change. Predicted probabilities for visiting ANC four or more times (ANC4+) and giving birth in a facility were derived using mixed-effects logistic regression models adjusted for ideational and sociodemographic variables.

\section{Results}

Among 3,039 women, 23.6\% (95\% Cl: 18.0\%-30.3\%) attended ANC4+ times and 15.5\% (95\% Cl: $11.8 \%-20.1 \%$ ) gave birth in a facility. Among women who did not attend ANC4+ times or have a facilitybased delivery during their last pregnancies, the most commonly cited reasons were lack of perceived need ( $42 \%$ and $67 \%$, respectively) and spousal opposition ( $25 \%$ and $27 \%$, respectively). Women who knew any ANC benefit or the recommended number of ANC visits were 3.2- and 2.1-times more likely to attend ANC4+ times. Women who held positive views about health facilities for childbirth had 1.2- and 2.6-times higher likelihood of attending ANC4+ times and facility delivery, while women who believed ANC was only for sickness or pregnancy complications had $17 \%$ lower likelihood of attending ANC4+ times. Self-efficacy and supportive spousal influence were also significantly associated with both outcomes.

\section{Conclusions}

To improve pregnancy and childbirth practices in northwestern Nigeria, programs should address a range of psychosocial factors - across cognitive, emotional and social domains - that were significantly associated with pregnancy and childbirth behaviors: raising knowledge and dispelling myths, building women's confidence to access services, engaging spousal support in decision-making, and improving perceived (and actual) maternal health services quality.

\section{Background}

Globally, there are an estimated 300,000 maternal deaths each year, and approximately $20 \%$ of these deaths occur in Nigeria alone.(1) In 2015, it was estimated that Nigeria's maternal mortality ratio (MMR) was 800 deaths per 100,000 live births, which totals approximately 58,000 deaths.(1) It is likely that the 
MMR is much higher in northwestern Nigeria where maternal health outcomes are typically worse than national averages.(2) Given the burden of maternal mortality in Nigeria, improving maternal health in this country is both a national priority and a cornerstone for achieving the United Nations Sustainable Development Goals.(3-5)

Antenatal care (ANC) visits and facility-based deliveries are critical contact points with the formal health system that present opportunities to reach mothers and newborns with effective interventions to improve their chances of survival and well-being.(6) The World Health Organization (WHO) has recommended at least four antenatal care visits (ANC4+) during pregnancy, and more recently eight routine check-ups, in order to provide a range of interventions for a positive pregnancy experience.(6) WHO also promotes giving birth in a health facility with skilled personnel, since most deaths are due to direct obstetric causes that occur around the time of delivery and are difficult to predict in advance.(7) Nigeria national guidelines for pregnancy and childbirth are closely aligned with these global recommendations.(8)

Yet, despite the importance of these essential maternal health services, there remains low utilization of ANC and facility-based delivery in northwestern Nigeria.(9) According to the 2018 Nigeria Demographic and Health Survey (DHS), only half (53\%) of women aged 15-49 years in northwestern Nigeria attended ANC at least one time with a skilled provider during her last pregnancy, and only $16 \%$ gave birth in a facility.(2) Previous qualitative and quantitative research conducted in northwestern Nigeria suggests that barriers to uptake of these services may include distance to the health facility, healthcare costs, spousal disapproval, religious beliefs, maternal education, lack of perceived need, as well as broader gender norms and sociocultural dynamics.(10-15)

Psychosocial factors are also posited by theories as important intermediate determinants of a person's actions, and are often targeted by social and behavioral change (SBC) programs to spur action.(16) In the Ideation Model of Strategic Communication and Behavior Change, psychosocial factors are theorized to consist of three ideational domains - cognitive, emotional and social - and each domain consists of specific behavioral influences including: knowledge, attitudes, beliefs, perceived risk, subjective norms and self-image (cognitive); emotional response, empathy, and self-efficacy (emotional); and social support, social influence, interpersonal communication and personal advocacy (social).

Yet, despite the theorized importance of psychosocial influences on behavioral outcomes, there remains limited quantitative research that examines their relationship with pregnancy and childbirth behaviors in low- and middle-income countries (LMICs) including Nigeria. In this paper, we aimed to use this behavioral change model to develop a set of pregnancy-related ideational metrics relevant to the northwestern Nigerian context, and to subsequently examine their associations with the use of essential maternal health services including attending ANC4 + times and giving birth in a facility.

\section{Methods}

\section{Study setting}


This study was conducted in Kebbi, Sokoto, and Zamfara States in northwestern Nigeria within wards targeted for a social and behavior change (SBC) program to help improve the health and well-being of women and children.(17) This study analyzed the baseline survey from an evaluation of this SBC program with data collected prior to initiation of the current SBC program activities.(18)

The three states share a border with Niger Republic, while Kebbi also borders Benin to the west. The estimated populations of these states were 4.9 million (Sokoto), 4.3 million (Kebbi), and 4.4 million (Zamfara) in 2015.(19) Hausa is the dominant ethnicity and Islam is the main religion. The study area also has some of the highest poverty rates within Nigeria, and among the worst maternal and child mortality rates. According to the 2018 Nigeria Demographic and Health Survey,(2) the under-five mortality rate was highest in the northwest region at 187 deaths per 1000 live births. This same survey also showed that women's empowerment measures in the northwestern region were among the lowest nationally including: women who completed primary school (9\%), women who were unemployed in the past 12 months (45\%), women who owned a mobile phone (35\%) and married women who participated in her own healthcare decisions (20\%).

\section{Study design}

We conducted a two-stage cluster-sample cross-sectional survey of women with a child under 2 years living in Kebbi, Sokoto, and Zamfara States and within wards targeted for SBC programming. The survey sample size was determined based on the SBC program evaluation design, with three comparison groups. Sample size estimation allowed for a $10 \%$ non-response rate, a power criterion of 0.80 , an alpha coefficient of 0.05 , and varying intra-cluster correlations and minimal detectable differences for priority outcomes of the evaluation. Based on this estimation, a sample size of approximately 3,000 women with a child under 2 years was estimated for survey inclusion. At the first sampling stage, we selected 108 enumeration areas (EAs) from program wards within the three states (36 EAs per state) using digital maps and a grid sampling methodology. At the second sampling stage, all households within each sampled EA were enumerated to randomly select households with a resident woman aged 15-49 years who had a child under 2 years.

\section{Data collection}

Fieldwork was conducted in September-October 2019 over a 4-week period. Interviewer training occurred during the 1-week period prior to data collection. This training reviewed the study objectives, protocol and instruments, fieldwork procedures and ethical considerations. All interviewers participated in a pilot exercise that tested skip patterns, checked questionnaire translation (Hausa), and assessed question appropriateness and sequence. There were two questionnaires administered to survey participants. The household questionnaire collected information on household members, characteristics and assets. The female questionnaire collected information on respondent demographics, reproductive history, and gender norms. For women with a child under 2 years, respondents were specifically asked about behaviors during their last pregnancy in a standardized format to the Nigeria Demographic and Health Survey.(2) These behaviors included ANC attendance, timing, location, provider type, content and number of check-ups during her last pregnancy. Women were also asked where she gave birth to her youngest child and who assisted with the delivery. Women who did not attend ANC or who gave birth outside a facility were asked 
about reasons for service non-use. In addition, all respondents were asked ideational questions related to various health behaviors, including pregnancy and childbirth, which are described in the next section.

\section{Explanatory variables}

Table 1 presents the definition of pregnancy-related ideations that were the main explanatory variables in this analysis. Ideational questions were developed using Kincaid's Model of Strategic Communication and Behavior Change, and as appropriate, adapted from ideational research in other health areas. $(16,20-22)$ Sociodemographic variables were included in the analysis based on empirical evidence of their relationship with use of maternal health services in Nigeria and elsewhere. $(13,23-25)$ These variables included maternal age (15-24, 25-34, or 35-49 years old), maternal education (any formal education, or none or informal education), spousal education (any formal education, or none or informal education), maternal employment (works outside home or student, or does not work outside home), spousal employment (works outside home or student, or does not work outside home), and household wealth (lowest, second, third, fourth or highest wealth quintile). Wealth was measured using an asset-based measure constructed from ownership of key consumer durables and then compiled into an index using principal components analysis. (26) Households were then categorized into quintiles from lowest to highest wealth asset ranking. 
Table 1

Pregnancy-related ideational metrics

\begin{tabular}{|c|c|c|c|}
\hline Dimension & Domain & $\begin{array}{l}\text { Likert-scale statement or } \\
\text { question }\end{array}$ & Response coding for analyses \\
\hline \multirow[t]{8}{*}{ COGNITIVE } & \multirow[t]{3}{*}{ Knowledge } & $\begin{array}{l}\text { How many times should a } \\
\text { woman receive a check-up } \\
\text { during pregnancy? }\end{array}$ & $\begin{array}{l}\text { Spontaneously reports } 4 \text { or more times } \\
\text { versus other responses }\end{array}$ \\
\hline & & $\begin{array}{l}\text { In your opinion, when should } \\
\text { a pregnant woman first go to } \\
\text { ANC? }\end{array}$ & $\begin{array}{l}\text { Spontaneously reports "as soon as she } \\
\text { thinks she is pregnant" or "first } \\
\text { trimester" versus other responses }\end{array}$ \\
\hline & & $\begin{array}{l}\text { What are some benefits to } \\
\text { the mother of ANC } \\
\text { attendance? }\end{array}$ & $\begin{array}{l}\text { Spontaneously reports any benefit } \\
\text { versus other responses }\end{array}$ \\
\hline & \multirow[t]{3}{*}{$\begin{array}{l}\text { Beliefs } \\
\text { about } \\
\text { pregnancy }\end{array}$} & $\begin{array}{l}\text { Pregnant women attending } \\
4+\text { ANC visits have safer } \\
\text { pregnancies }\end{array}$ & $\begin{array}{l}\text { Agree (strongly or somewhat) versus } \\
\text { disagree (strongly or somewhat) versus } \\
\text { don't know }\end{array}$ \\
\hline & & $\begin{array}{l}\text { Pregnant women need ANC } \\
\text { only when sick }\end{array}$ & $\begin{array}{l}\text { Agree (strongly or somewhat) versus } \\
\text { disagree (strongly or somewhat) versus } \\
\text { don't know }\end{array}$ \\
\hline & & $\begin{array}{l}\text { Only first-time pregnant } \\
\text { women need ANC }\end{array}$ & $\begin{array}{l}\text { Agree (strongly or somewhat) versus } \\
\text { disagree (strongly or somewhat) versus } \\
\text { don't know }\end{array}$ \\
\hline & \multirow[t]{2}{*}{$\begin{array}{l}\text { Beliefs } \\
\text { about } \\
\text { health } \\
\text { services }\end{array}$} & $\begin{array}{l}\text { The health facility is the best } \\
\text { place to deliver a baby }\end{array}$ & $\begin{array}{l}\text { Agree (strongly or somewhat) versus } \\
\text { disagree (strongly or somewhat) versus } \\
\text { don't know }\end{array}$ \\
\hline & & $\begin{array}{l}\text { It is better to use a traditional } \\
\text { provider than a health facility } \\
\text { for ANC }\end{array}$ & $\begin{array}{l}\text { Agree (strongly or somewhat) versus } \\
\text { disagree (strongly or somewhat) versus } \\
\text { don't know }\end{array}$ \\
\hline \multirow[t]{3}{*}{ EMOTIONAL } & \multirow[t]{3}{*}{$\begin{array}{l}\text { Self- } \\
\text { efficacy }\end{array}$} & $\begin{array}{l}\text { Confidence that you could } \\
\text { get to a health facility for } \\
\text { ANC }\end{array}$ & \multirow[t]{2}{*}{$\begin{array}{l}\text { Confident (strongly or somewhat) } \\
\text { versus uncertain (strongly or somewhat) } \\
\text { versus don't know }\end{array}$} \\
\hline & & $\begin{array}{l}\text { Confidence that you could } \\
\text { get to a health facility for } \\
\text { delivery }\end{array}$ & \\
\hline & & $\begin{array}{l}\text { Confidence to start a } \\
\text { conversation with husband } \\
\text { about ANC Confidence to } \\
\text { start a conversation with } \\
\text { husband about facility } \\
\text { delivery }\end{array}$ & $\begin{array}{l}\text { Confident (strongly or somewhat) } \\
\text { versus uncertain (strongly or somewhat) } \\
\text { versus don't know }\end{array}$ \\
\hline \multirow[t]{2}{*}{ SOCIAL } & \multirow[t]{2}{*}{$\begin{array}{l}\text { Social } \\
\text { influence }\end{array}$} & $\begin{array}{l}\text { Who else influences your } \\
\text { decision about going to } 4+ \\
\text { ANC visits }\end{array}$ & \multirow{2}{*}{$\begin{array}{l}\text { Spontaneously reports partner versus } \\
\text { other responses; spontaneously reports } \\
\text { mother-in-law versus other responses; } \\
\text { spontaneously reports health worker } \\
\text { versus other responses }\end{array}$} \\
\hline & & $\begin{array}{l}\text { Who else influences your } \\
\text { decision about having a } \\
\text { facility delivery }\end{array}$ & \\
\hline
\end{tabular}




\begin{tabular}{|llll|}
\hline Dimension & Domain & $\begin{array}{l}\text { Likert-scale statement or } \\
\text { question }\end{array}$ & Response coding for analyses \\
\hline & $\begin{array}{l}\text { Social } \\
\text { norms }\end{array}$ & $\begin{array}{l}\text { It's important for a woman to } \\
\text { discuss her pregnancy with } \\
\text { her husband }\end{array}$ & $\begin{array}{l}\text { Agree (strongly or somewhat) versus } \\
\text { disagree (strongly or somewhat) versus } \\
\text { don't know }\end{array}$ \\
\hline
\end{tabular}

\section{Outcome definitions}

There were two main outcomes. First, ANC4 + was defined as reported attendance at ANC at least 4 times by the respondent during her last pregnancy within the past two years, and where the first visit was with a skilled provider (doctor, nurse or midwife). Specifically, during the survey interview, the woman was asked if she saw anyone for ANC during her last pregnancy, and if so, whom did she see for the first visit. She was then asked how many antenatal check-ups she had during this pregnancy. Second, facility-based delivery was defined as giving birth in a health facility during her last pregnancy within the past two years. During the survey interview, the woman was asked where she gave birth during this pregnancy, and a facility-based delivery included: government hospital, primary health care center (PHC), health post, community health outreach post, nursing/maternity home, private hospital or private clinic.

\section{Data analysis}

Mixed-effects logistic regression models were used to estimate average marginal effects, or the change in the probability of an outcome when a predictor variable increased by one unit. For binary predictor variables, marginal effects represent the additional likelihood of using antenatal care or delivering in a facility for a person who possesses that characteristic (e.g., husband influences decisions about delivering in a facility) relative to a person who does not. All ideational and sociodemographic variables were included in the models as categorical fixed effects nested within cluster identifiers. We tested for multicollinearity among variables using variance inflation factors. The level of statistical significance was set to 0.05 . Point estimates were tabulated using weights to account for unequal probabilities of selection. Standard error estimation accounted for data clustering in the complex survey design. All analyses were conducted in Stata 16 (STATA Corp, College Station, TX).

\section{Results}

A total of 3,039 women responded to questions about the antenatal and delivery care received during her last completed pregnancy in the past two years. Among these respondents, $23.6 \%$ (95\% Cl: 18.0-30.3) made ANC4 + visits during her last pregnancy, and 15.5\% (95\% Cl: 11.8-20.1) gave birth in a health facility (Table 2). The mean age of respondents in the sample was 26.0 years, while the average spousal age was 33.3 years. Only $26.1 \%$ and $31.0 \%$ of respondents and their spouses had any formal schooling, while $50.3 \%$ and $91.9 \%$ worked outside the home, respectively. There was a mean of 2.2 wives per husband in the study sample. 
Table 2

Study sample

\begin{tabular}{|lllll|}
\hline & Kebbi & Sokoto & Zamfara & Total \\
\hline & $\%$ & $\%$ & $\%$ & $\%$ \\
\hline Respondent demographics & $\mathrm{N}=892$ & $\mathrm{~N}=1078$ & $\mathrm{~N}=1069$ & $\mathrm{~N}=3039$ \\
\hline Age in years (mean) & & & & \\
\hline Any formal schooling, primary attendance or higher & 25.2 & 19.3 & 28.9 & 26.1 \\
\hline Employment outside home or student & 47.9 & 45.6 & 52.8 & 50.3 \\
\hline ANC4 + attendance during last pregnancy & 23.6 & 17.0 & 26.1 & 23.6 \\
\hline Facility-based delivery during last pregnancy & 14.8 & 13.8 & 16.3 & 15.5 \\
\hline Spousal demographics & & & & \\
\hline Age in years (mean) & 25.3 & 29.0 & 37.3 & 33.3 \\
\hline Any formal schooling, primary attendance or higher & 27.3 & 19.4 & 36.5 & 31.0 \\
\hline Employment outside home or student & 93.7 & 93.2 & 90.9 & 91.9 \\
\hline Number of wives (mean) & 2.1 & 2.0 & 2.2 & 2.2 \\
\hline
\end{tabular}

\section{Bivariate results of ANC4 + and facility-based delivery by ideational variables}

Among respondents attending ANC4 + times during her last pregnancy, a greater percentage had positive cognitive, emotional or social ideations than negative or disagreeable ones. For example, $48.1 \%$ (95\% Cl: $39.2 \%-57.2 \%$ ) of women who attended ANC4 + times during her last pregnancy knew that women should receive four or more check-ups during pregnancy compared to $4.9 \%$ (95\% Cl: $3.2 \%-7.5 \%)$ who were unaware (knowledge) (Table 3). Similarly, 11.7\% (95\% Cl: 23.3\%-40.6\%) believed pregnant women need ANC only when sick compared to $34.9 \%$ (95\% Cl: $26.4 \%-44.4 \%$ ) who did not hold this belief (beliefs). At the same time, $34.0 \%$ (95\% Cl: $26.6 \%-42.3 \%$ ) of these respondents were confident they could get to a health facility for ANC compared to $1.3 \%$ (95\% Cl: $0.6 \%-3.1 \%$ ) who were uncertain (self-efficacy). Among respondents attending ANC4 + times during their last pregnancy, health workers (37.9\%, 95\% Cl: $20.1 \%-59.6 \%$ ) and spouses/partners (28.9\%, $95 \% \mathrm{Cl}: 21.5 \%-37.5 \%)$ were most commonly cited as influencing the woman's decision. 
Table 3

Characteristics of ANC4 + attendance and facility-based delivery by sociodemographic and ideational variables

\begin{tabular}{|c|c|c|c|c|c|c|}
\hline & & $\begin{array}{l}N \text { women } \\
15-49 \text { years } \\
\text { with a live } \\
\text { birth in past } \\
2 \text { years }\end{array}$ & $\begin{array}{l}\% \\
\text { ANC4+ }\end{array}$ & $\begin{array}{l}(95 \% \\
\mathrm{Cl})\end{array}$ & $\begin{array}{l}\% \\
\text { facility } \\
\text { delivery }\end{array}$ & $\begin{array}{l}(95 \% \\
\mathrm{Cl})\end{array}$ \\
\hline Total & & 3,039 & 23.6 & $\begin{array}{l}18.0- \\
30.3\end{array}$ & 15.5 & $\begin{array}{l}11.8- \\
20.1\end{array}$ \\
\hline \multicolumn{7}{|l|}{ Ideational metrics } \\
\hline \multirow{2}{*}{$\begin{array}{l}\text { How many times should a } \\
\text { woman receive a check-up } \\
\text { during pregnancy? }\end{array}$} & $\begin{array}{l}4 \text { or more } \\
\text { times }\end{array}$ & 1,266 & 48.1 & $\begin{array}{l}39.2- \\
57.2\end{array}$ & 29.6 & $\begin{array}{l}23.1- \\
36.9\end{array}$ \\
\hline & Other response & 1,773 & 4.9 & $\begin{array}{l}3.2- \\
7.5\end{array}$ & 4.8 & $\begin{array}{l}3.2- \\
7.2\end{array}$ \\
\hline \multirow[t]{2}{*}{$\begin{array}{l}\text { In your opinion, when } \\
\text { should a pregnant woman } \\
\text { first go to ANC? }\end{array}$} & $\begin{array}{l}\text { As soon as she } \\
\text { thinks she is } \\
\text { pregnant or in } \\
\text { the first } \\
\text { trimester }\end{array}$ & 880 & 34.9 & $\begin{array}{l}26.7- \\
44.2\end{array}$ & 13.0 & $\begin{array}{l}9.2- \\
18.1\end{array}$ \\
\hline & Other response & 2,159 & 19.1 & $\begin{array}{l}13.2- \\
26.9\end{array}$ & 21.9 & $\begin{array}{l}16.0- \\
29.1\end{array}$ \\
\hline \multirow{2}{*}{$\begin{array}{l}\text { What are some benefits to } \\
\text { the mother of ANC } \\
\text { attendance? }\end{array}$} & Any benefit & 2,462 & 28.8 & $\begin{array}{l}22.2- \\
36.3\end{array}$ & 18.4 & $\begin{array}{l}14.1- \\
23.7\end{array}$ \\
\hline & Other response & 577 & 0.3 & $\begin{array}{l}0.1- \\
1.5\end{array}$ & 2.5 & $\begin{array}{l}0.1- \\
5.4\end{array}$ \\
\hline \multirow{3}{*}{$\begin{array}{l}\text { Agreed (strongly or } \\
\text { somewhat) that pregnant } \\
\text { women visiting ANC have } \\
\text { safer pregnancies }\end{array}$} & Agreed & 1,979 & 35.6 & $\begin{array}{l}28.4- \\
43.5\end{array}$ & 23.5 & $\begin{array}{l}18.5- \\
29.4\end{array}$ \\
\hline & Disagreed & 744 & 4.6 & $\begin{array}{l}2.6- \\
8.2\end{array}$ & 2.8 & $\begin{array}{l}1.2- \\
6.3\end{array}$ \\
\hline & Don't know & 316 & 1.2 & $\begin{array}{l}0.4- \\
3.5\end{array}$ & 0.9 & $\begin{array}{l}0.3- \\
3.0\end{array}$ \\
\hline \multirow{3}{*}{$\begin{array}{l}\text { Agreed (strongly or } \\
\text { somewhat) that pregnant } \\
\text { women need ANC only } \\
\text { when sick }\end{array}$} & Agreed & 1,268 & 11.7 & $\begin{array}{l}23.3- \\
40.6\end{array}$ & 12.7 & $\begin{array}{l}8.5- \\
18.6\end{array}$ \\
\hline & Disagreed & 1,569 & 34.9 & $\begin{array}{l}26.4- \\
44.4\end{array}$ & 19.2 & $\begin{array}{l}13.8- \\
26.1\end{array}$ \\
\hline & Don't know & 202 & 2.2 & $\begin{array}{l}5.5- \\
8.0\end{array}$ & 1.7 & $\begin{array}{l}0.4- \\
7.0\end{array}$ \\
\hline $\begin{array}{l}\text { Agreed (very or somewhat) } \\
\text { that only first-time }\end{array}$ & Agreed & 798 & 15.8 & $\begin{array}{l}9.6- \\
24.8\end{array}$ & 16.9 & $\begin{array}{l}11.2- \\
24.5\end{array}$ \\
\hline
\end{tabular}

(-) Not applicable. Variable not included in the regression model for that outcome. 


\begin{tabular}{|c|c|c|c|c|c|c|}
\hline & & $\begin{array}{l}N \text { women } \\
15-49 \text { years } \\
\text { with a live } \\
\text { birth in past } \\
2 \text { years }\end{array}$ & $\begin{array}{l}\% \\
\text { ANC4+ }\end{array}$ & $\begin{array}{l}(95 \% \\
\mathrm{Cl})\end{array}$ & $\begin{array}{l}\% \\
\text { facility } \\
\text { delivery }\end{array}$ & $\begin{array}{l}(95 \% \\
\mathrm{Cl})\end{array}$ \\
\hline & Disagreed & 1,949 & 29.8 & $\begin{array}{l}22.5- \\
38.3\end{array}$ & 17.0 & $\begin{array}{l}12.4- \\
22.8\end{array}$ \\
\hline & Don't know & 292 & 2.0 & $\begin{array}{l}0.7- \\
5.2\end{array}$ & 3.1 & $\begin{array}{l}1.2- \\
7.9\end{array}$ \\
\hline \multirow{3}{*}{$\begin{array}{l}\text { Agreed (very or somewhat) } \\
\text { that the health facility is } \\
\text { the best place to deliver a } \\
\text { baby }\end{array}$} & Agreed & 1,688 & 33.8 & $\begin{array}{l}26.3- \\
42.2\end{array}$ & 27.2 & $\begin{array}{l}21.3- \\
33.9\end{array}$ \\
\hline & Disagreed & 1,254 & 12.1 & $\begin{array}{l}7.9- \\
18.1\end{array}$ & 1.7 & $\begin{array}{l}0.9- \\
3.1\end{array}$ \\
\hline & Don't know & 97 & 1.0 & $\begin{array}{l}0.2- \\
4.0\end{array}$ & 1.1 & $\begin{array}{l}0.3- \\
4.3\end{array}$ \\
\hline \multirow{3}{*}{$\begin{array}{l}\text { Agreed (very or somewhat) } \\
\text { that it's better to use a } \\
\text { traditional provider than a } \\
\text { facility for ANC }\end{array}$} & Agreed & 1,104 & 12.0 & $\begin{array}{l}7.6- \\
18.2\end{array}$ & 10.2 & $\begin{array}{l}6.8- \\
14.8\end{array}$ \\
\hline & Disagreed & 1,752 & 32.1 & $\begin{array}{l}24.7- \\
40.5\end{array}$ & 19.9 & $\begin{array}{l}14.7- \\
26.4\end{array}$ \\
\hline & Don't know & 183 & 5.8 & $\begin{array}{l}2.1- \\
15.1\end{array}$ & 2.8 & $\begin{array}{l}1.3- \\
6.1\end{array}$ \\
\hline \multirow{3}{*}{$\begin{array}{l}\text { Agreed (strongly or } \\
\text { somewhat) that it's } \\
\text { important for a woman to } \\
\text { discuss her pregnancy with } \\
\text { her husband }\end{array}$} & Agreed & 2,784 & 24.8 & $\begin{array}{l}19.0- \\
31.8\end{array}$ & 16.5 & $\begin{array}{l}12.5- \\
21.4\end{array}$ \\
\hline & Disagreed & 184 & 8.9 & $\begin{array}{l}3.5- \\
20.7\end{array}$ & 3.5 & $\begin{array}{l}1.4- \\
8.6\end{array}$ \\
\hline & Don't know & 71 & 2.7 & $\begin{array}{l}0.5- \\
12.4\end{array}$ & 2.4 & $\begin{array}{l}0.9- \\
6.2\end{array}$ \\
\hline \multirow{3}{*}{$\begin{array}{l}\text { Confident (strongly or } \\
\text { somewhat) that she could } \\
\text { get to a health facility for } \\
\text { ANC [delivery] }\end{array}$} & Confident & 2,084 & 34.0 & $\begin{array}{l}26.6- \\
42.3\end{array}$ & 29.9 & $\begin{array}{l}23.7- \\
37.0\end{array}$ \\
\hline & Uncertain & 876 & 1.3 & $\begin{array}{l}0.6- \\
3.1\end{array}$ & 1.1 & $\begin{array}{l}0.6- \\
1.9\end{array}$ \\
\hline & Don't know & 79 & 0.9 & $\begin{array}{l}0.1- \\
7.1\end{array}$ & 0.3 & $\begin{array}{l}0.0- \\
2.9\end{array}$ \\
\hline \multirow{3}{*}{$\begin{array}{l}\text { Besides yourself, who else } \\
\text { influences your decision to } \\
\text { attend ANC [give birth] at a } \\
\text { facility? }\end{array}$} & Partner & 2,045 & 28.9 & $\begin{array}{l}21.5- \\
37.5\end{array}$ & 21.8 & $\begin{array}{l}16.3^{-} \\
28.5\end{array}$ \\
\hline & Other response & 994 & 12.9 & $\begin{array}{l}8.3- \\
19.4\end{array}$ & 6.8 & $\begin{array}{l}4.5- \\
10.2\end{array}$ \\
\hline & Mother-in-law & 66 & 20.6 & $\begin{array}{l}9.3- \\
39.5\end{array}$ & 13.5 & $\begin{array}{l}6.5- \\
26.1\end{array}$ \\
\hline
\end{tabular}

(-) Not applicable. Variable not included in the regression model for that outcome. 


\begin{tabular}{|c|c|c|c|c|c|c|}
\hline & & $\begin{array}{l}N \text { women } \\
15-49 \text { years } \\
\text { with a live } \\
\text { birth in past } \\
2 \text { years }\end{array}$ & $\begin{array}{l}\% \\
\text { ANC4+ }\end{array}$ & $\begin{array}{l}(95 \% \\
\mathrm{Cl})\end{array}$ & $\begin{array}{l}\% \\
\text { facility } \\
\text { delivery }\end{array}$ & $\begin{array}{l}(95 \% \\
\mathrm{Cl})\end{array}$ \\
\hline & Other response & 2,973 & 23.6 & $\begin{array}{l}18.0- \\
30.4\end{array}$ & 15.6 & $\begin{array}{l}11.8- \\
20.2\end{array}$ \\
\hline & Health worker & 66 & 37.9 & $\begin{array}{l}20.1- \\
59.6\end{array}$ & 15.1 & $\begin{array}{l}11.4- \\
19.6\end{array}$ \\
\hline & Other response & 2,973 & 23.2 & $\begin{array}{l}17.6- \\
30.0\end{array}$ & 29.6 & $\begin{array}{l}15.3- \\
49.4\end{array}$ \\
\hline \multirow{3}{*}{$\begin{array}{l}\text { Likely (very or somewhat) } \\
\text { to make at least four or } \\
\text { more ANC visits [give birth } \\
\text { in a facility] during her next } \\
\text { pregnancy }\end{array}$} & Likely & 2,034 & 33.8 & $\begin{array}{l}26.5- \\
42.1\end{array}$ & 29.0 & $\begin{array}{l}22.9- \\
36.0\end{array}$ \\
\hline & Unlikely & 865 & 3.2 & $\begin{array}{l}1.5- \\
6.8\end{array}$ & 1.3 & $\begin{array}{l}0.7- \\
2.4\end{array}$ \\
\hline & Don't know & 140 & 2.7 & $\begin{array}{l}0.8- \\
8.7\end{array}$ & 0.9 & $\begin{array}{l}0.2- \\
4.0\end{array}$ \\
\hline \multicolumn{7}{|l|}{ Socio-demographic variables } \\
\hline \multirow[t]{5}{*}{ Household wealth quintile } & Lowest & 716 & 7.7 & $\begin{array}{l}4.4- \\
13.0\end{array}$ & 4.8 & $\begin{array}{l}2.9- \\
8.0\end{array}$ \\
\hline & Second & 604 & 14.2 & $\begin{array}{l}10.0- \\
19.8\end{array}$ & 8.0 & $\begin{array}{l}5.7- \\
11.2\end{array}$ \\
\hline & Middle & 600 & 16.9 & $\begin{array}{l}11.8- \\
23.5\end{array}$ & 11.0 & $\begin{array}{l}7.4- \\
16.0\end{array}$ \\
\hline & Fourth & 496 & 27.1 & $\begin{array}{l}18.1- \\
38.5\end{array}$ & 13.4 & $\begin{array}{l}8.8- \\
20.0\end{array}$ \\
\hline & Highest & 623 & 52.8 & $\begin{array}{l}42.4- \\
62.9\end{array}$ & 40.7 & $\begin{array}{l}31.3- \\
50.8\end{array}$ \\
\hline \multirow[t]{2}{*}{ Maternal education } & $\begin{array}{l}\text { Any formal } \\
\text { education }\end{array}$ & 499 & 59.5 & $\begin{array}{l}51.3- \\
67.2\end{array}$ & 43.9 & $\begin{array}{l}34.3^{-} \\
53.9\end{array}$ \\
\hline & $\begin{array}{l}\text { None or } \\
\text { informal }\end{array}$ & 2,540 & 16.9 & $\begin{array}{l}12.6- \\
22.3\end{array}$ & 10.3 & $\begin{array}{l}7.9- \\
13.3\end{array}$ \\
\hline \multirow[t]{3}{*}{ Maternal age } & $15-24$ years & 1,275 & 19.0 & $\begin{array}{l}14.1- \\
25.2\end{array}$ & 14.4 & $\begin{array}{l}10.6^{-} \\
19.3^{-}\end{array}$ \\
\hline & $25-34$ years & 1,376 & 25.4 & $\begin{array}{l}19.0- \\
33.1\end{array}$ & 15.7 & $\begin{array}{l}11.6- \\
21.0\end{array}$ \\
\hline & $35-49$ years & 388 & 31.3 & $\begin{array}{l}22.5- \\
41.7\end{array}$ & 18.4 & $\begin{array}{l}13.1- \\
25.3\end{array}$ \\
\hline
\end{tabular}

(-) Not applicable. Variable not included in the regression model for that outcome. 


\begin{tabular}{|c|c|c|c|c|c|c|}
\hline & & $\begin{array}{l}N \text { women } \\
15-49 \text { years } \\
\text { with a live } \\
\text { birth in past } \\
2 \text { years }\end{array}$ & $\begin{array}{l}\% \\
\text { ANC4+ }\end{array}$ & $\begin{array}{l}(95 \% \\
\mathrm{Cl})\end{array}$ & $\begin{array}{l}\% \\
\text { facility } \\
\text { delivery }\end{array}$ & $\begin{array}{l}(95 \% \\
\mathrm{Cl})\end{array}$ \\
\hline \multirow[t]{4}{*}{ Maternal occupation } & $\begin{array}{l}\text { Work outside } \\
\text { home or } \\
\text { student }\end{array}$ & 1,469 & 24.1 & $\begin{array}{l}17.1- \\
32.8\end{array}$ & 13.0 & $\begin{array}{l}9.4- \\
17.9\end{array}$ \\
\hline & $\begin{array}{l}\text { No work } \\
\text { outside home }\end{array}$ & 1,409 & 23.9 & $\begin{array}{l}17.0- \\
32.6\end{array}$ & 19.2 & $\begin{array}{l}13.5- \\
26.5\end{array}$ \\
\hline & Other & 111 & 11.5 & $\begin{array}{l}3.7- \\
30.3\end{array}$ & 10.9 & $\begin{array}{l}4.5- \\
24.2\end{array}$ \\
\hline & Missing & 50 & - & - & - & - \\
\hline \multirow[t]{4}{*}{ Spousal occupation } & $\begin{array}{l}\text { Work outside } \\
\text { home or } \\
\text { student }\end{array}$ & 2,742 & 23.5 & $\begin{array}{l}17.7- \\
30.4\end{array}$ & 15.8 & $\begin{array}{l}11.9- \\
20.6\end{array}$ \\
\hline & $\begin{array}{l}\text { No work } \\
\text { outside home }\end{array}$ & 149 & 17.4 & $\begin{array}{l}10.1- \\
28.3\end{array}$ & 9.8 & $\begin{array}{l}4.7- \\
19.4\end{array}$ \\
\hline & Other & 98 & 32.2 & $\begin{array}{l}17.6- \\
51.3\end{array}$ & 22.4 & $\begin{array}{l}11.1- \\
39.8\end{array}$ \\
\hline & Missing & 50 & & & & \\
\hline \multirow[t]{3}{*}{ Spousal education } & $\begin{array}{l}\text { Any formal } \\
\text { education }\end{array}$ & 2,056 & 12.6 & $\begin{array}{l}9.5- \\
16.6\end{array}$ & 7.7 & $\begin{array}{l}5.9- \\
10.2\end{array}$ \\
\hline & $\begin{array}{l}\text { None or } \\
\text { informal }\end{array}$ & 933 & 47.6 & $\begin{array}{l}38.6- \\
56.8\end{array}$ & 33.5 & $\begin{array}{l}25.9- \\
42.1\end{array}$ \\
\hline & Missing & 50 & - & - & - & - \\
\hline
\end{tabular}

A similar ideational pattern occurred among women who gave birth in a facility during her last pregnancy. Specifically, $29.6 \%$ (95\% Cl: $23.1 \%-36.9 \%$ ) of respondents who gave birth in a facility knew that women should receive four or more check-ups during pregnancy compared to $4.8 \%$ (95\% Cl: $3.2 \%-7.2 \%$ ) of these respondents who did not know (knowledge). Similarly, 27.2\% (95\% Cl: 21.3\%-33.9\%) of these same respondents believed that the health facility is the best place to deliver a baby compared to $1.7 \%(95 \% \mathrm{Cl}$ : 0.9\%-3.1\%) who disagreed (beliefs), while $29.9 \%$ (95\% Cl: $23.7 \%-37.0 \%)$ of these respondents were confident they could get to a health facility for delivery compared to $1.1 \%$ (95\% Cl: $0.6 \%-1.9 \%)$ who were uncertain (self-efficacy). Among respondents who gave birth at a facility during the last pregnancy, spouses/partners (21.8\%, 95\% Cl: $16.3 \%-28.5 \%)$ were most commonly cited as influencing the woman's decision to give birth in a facility.

Among women who did not attend ANC4 + times or give birth in a facility during the last pregnancy (Fig. 1), $41.4 \%$ and $66.8 \%$ of respondents did not perceive it as necessary to go to antenatal care $(95 \% \mathrm{Cl}$ : $33.0 \%-50.3 \%)$ or to give birth in a facility (95\% Cl: $58.5 \%-74.3 \%)$, respectively. Spousal opposition was also 
a commonly cited reason for not attending ANC (25.2\%, 95\% Cl: $18.3 \%-33.6 \%)$ or for giving birth in a facility (27.3\%, 95\% Cl: 20.8\%-34.9\%).

\section{Ideational associations with ANC4 + visits}

The psychosocial factors significantly associated with attending ANC4 + times in regression analyses included: ANC knowledge, beliefs about ANC efficacy and health services quality, self-efficacy and supportive spousal influence on decision-making (Table 4, Fig. 2). In the adjusted analysis, the probability of attending ANC4 + times increased 16 percentage points (95\% Cl: $0.12-0.19, p<0.001)$ if the respondent knew that women should receive four or more check-ups during pregnancy compared to those who were not aware (knowledge); increased 5 points ( $95 \% \mathrm{Cl}: 0.02-0.08, p=0.002)$ if she knew that women should initiate ANC in the first trimester or as soon as she thinks she is pregnant (knowledge); increased 16 points ( $95 \%$ Cl: $0.10-0.23, p<0.001)$ if she is able to report any ANC benefit for herself (knowledge); increased 5 points ( $95 \% \mathrm{Cl}: 0.01-0.10, \mathrm{p}=0.021)$ if she believed women who attend ANC4 + have safer pregnancies (beliefs); decreased 4 points ( $95 \% \mathrm{Cl}: 0.00-0.08, \mathrm{p}=0.037$ ) if she believed only sick pregnant women need ANC (beliefs); increased 4 percentage points $(95 \% \mathrm{Cl}: 0.01-0.08, p=0.006)$ if she believed that the health facility is the best place to deliver a baby (beliefs); increased 15 points $(95 \% \mathrm{Cl}: 0.11-0.20, p<0.001)$ if she felt confident that she could get to a health facility for ANC (self-efficacy); increased 4 points ( $95 \% \mathrm{Cl}$ : 0.00 $0.07, p=0.029$ ) if she reported that her spouse/partner supported her decision to attend ANC4+ (social influence). The following sociodemographic variables were also significantly associated with ANC4 + attendance in the adjusted analysis: household wealth, maternal employment, maternal education, and spousal education. 
Table 4

Ideational and sociodemographic associations with ANC4 + and facility-based delivery

ANC4+

Likert-scale
statement
or question

Ideational variables

\begin{tabular}{|c|c|c|c|c|c|c|c|c|c|}
\hline $\begin{array}{l}\text { How many } \\
\text { times }\end{array}$ & $\begin{array}{l}4 \text { or more } \\
\text { check-ups }\end{array}$ & 0.16 & 0.12 & 0.19 & $\begin{array}{l}< \\
0.001\end{array}$ & 0.06 & 0.03 & 0.09 & $\begin{array}{l}< \\
0.001\end{array}$ \\
\hline $\begin{array}{l}\text { receive a } \\
\text { check-up } \\
\text { during } \\
\text { pregnancy? }\end{array}$ & $\begin{array}{l}\text { Other } \\
\text { response }\end{array}$ & - & - & - & - & - & - & - & - \\
\hline
\end{tabular}

\begin{tabular}{llllllllll}
$\begin{array}{l}\text { In your } \\
\text { opinion, } \\
\text { when } \\
\text { should a }\end{array}$ & 0.05 & 0.02 & 0.08 & 0.002 & 0.01 & -0.02 & 0.04 & 0.626 \\
$\begin{array}{l}\text { shegnant } \\
\text { woman first } \\
\text { go to ANC? }\end{array}$ & $\begin{array}{l}\text { Other } \\
\text { response }\end{array}$ & - & - & - & - & - & - & - & - \\
\hline
\end{tabular}

\begin{tabular}{|c|c|c|c|c|c|c|c|c|c|}
\hline \multirow{2}{*}{$\begin{array}{l}\text { What are } \\
\text { some } \\
\text { benefits to } \\
\text { the mother } \\
\text { of ANC } \\
\text { attendance? }\end{array}$} & $\begin{array}{l}\text { Any } \\
\text { benefit } \\
\text { reported }\end{array}$ & 0.16 & 0.10 & 0.23 & $\begin{array}{l}< \\
0.001\end{array}$ & 0.01 & -0.05 & 0.08 & 0.671 \\
\hline & $\begin{array}{l}\text { Other } \\
\text { response }\end{array}$ & - & - & - & - & - & - & - & - \\
\hline
\end{tabular}

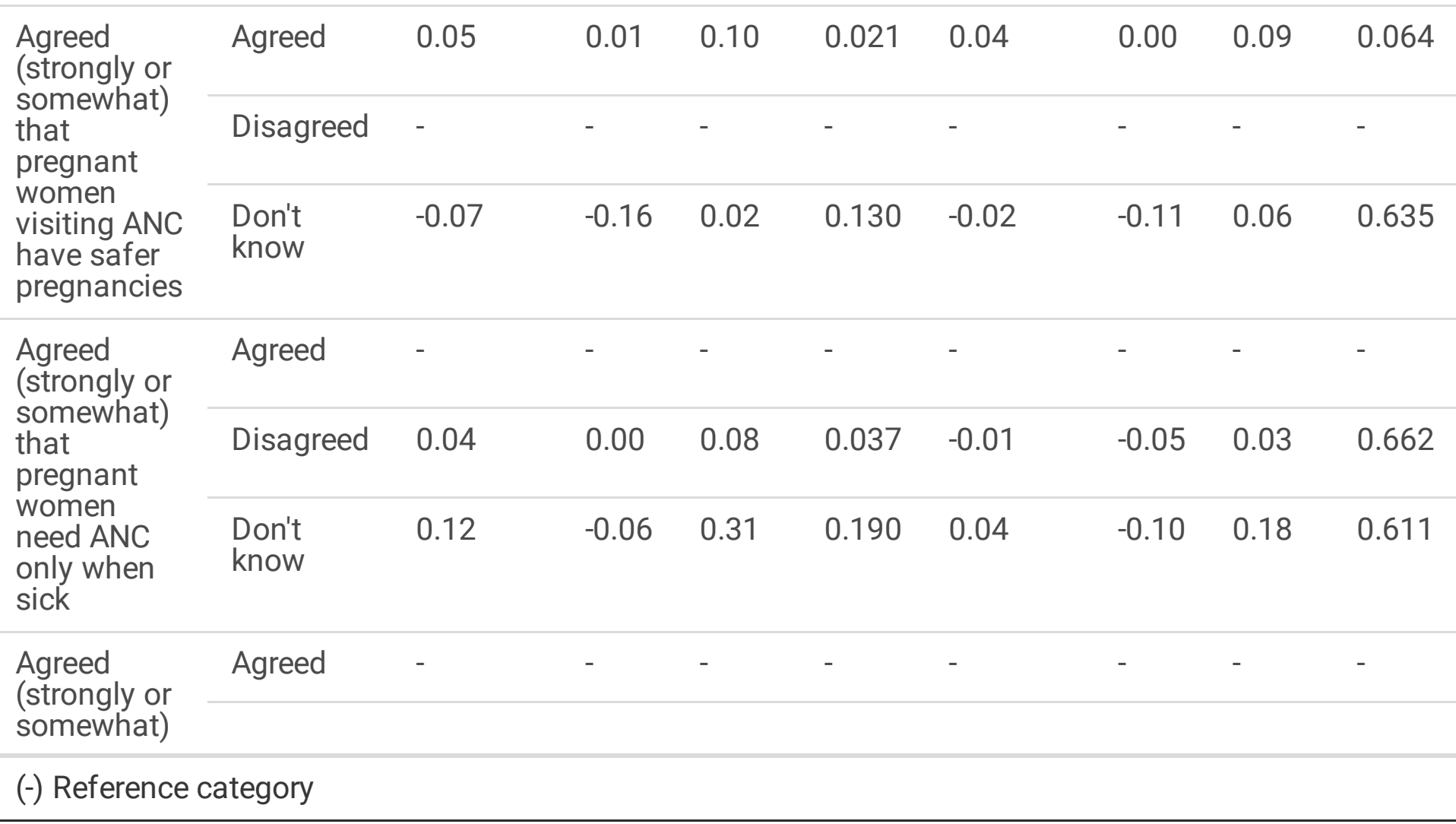




\begin{tabular}{|c|c|c|c|c|c|c|c|c|c|}
\hline \multirow{3}{*}{$\begin{array}{l}\text { that only } \\
\text { first-time } \\
\text { pregnant } \\
\text { women } \\
\text { need ANC }\end{array}$} & \multicolumn{3}{|c|}{ ANC4+ } & \multicolumn{6}{|c|}{ Facility-based delivery } \\
\hline & Disagreed & 0.03 & -0.01 & 0.08 & 0.129 & -0.01 & -0.05 & 0.03 & 0.592 \\
\hline & $\begin{array}{l}\text { Don't } \\
\text { know }\end{array}$ & -0.01 & -0.14 & 0.12 & 0.909 & 0.04 & -0.07 & 0.15 & 0.519 \\
\hline \multirow{3}{*}{$\begin{array}{l}\text { Agreed } \\
\text { (strongly or } \\
\text { somewhat) } \\
\text { that the } \\
\text { health } \\
\text { facility is } \\
\text { the best } \\
\text { place to } \\
\text { deliver a } \\
\text { baby }\end{array}$} & Agreed & 0.04 & 0.01 & 0.08 & 0.006 & 0.12 & 0.09 & 0.15 & $\begin{array}{l}<.001 \\
0.00\end{array}$ \\
\hline & Disagreed & - & - & - & - & - & - & - & - \\
\hline & $\begin{array}{l}\text { Don't } \\
\text { know }\end{array}$ & -0.04 & -0.22 & 0.14 & 0.643 & 0.08 & -0.06 & 0.23 & 0.276 \\
\hline \multirow{3}{*}{$\begin{array}{l}\text { Agreed } \\
\text { (strongly or } \\
\text { somewhat) } \\
\text { that it's } \\
\text { better to use } \\
\text { a traditional } \\
\text { provider } \\
\text { than a } \\
\text { facility for } \\
\text { ANC }\end{array}$} & Agreed & - & - & - & - & - & - & - & - \\
\hline & Disagreed & 0.01 & -0.02 & 0.04 & 0.467 & 0.04 & 0.01 & 0.07 & 0.016 \\
\hline & $\begin{array}{l}\text { Don't } \\
\text { know }\end{array}$ & 0.10 & 0.00 & 0.19 & 0.041 & 0.05 & -0.04 & 0.14 & 0.255 \\
\hline \multirow{3}{*}{$\begin{array}{l}\text { Agreed } \\
\text { (strongly or } \\
\text { somewhat) } \\
\text { that it's } \\
\text { important } \\
\text { for a } \\
\text { woman to } \\
\text { discuss her } \\
\text { pregnancy } \\
\text { with her } \\
\text { husband }\end{array}$} & Agreed & 0.01 & -0.06 & 0.08 & 0.767 & -0.04 & -0.11 & 0.04 & 0.343 \\
\hline & Disagreed & - & - & - & - & - & - & - & - \\
\hline & $\begin{array}{l}\text { Don't } \\
\text { know }\end{array}$ & 0.03 & -0.19 & 0.25 & 0.794 & 0.12 & -0.06 & 0.31 & 0.202 \\
\hline \multirow{3}{*}{$\begin{array}{l}\text { Confident } \\
\text { (strongly or } \\
\text { somewhat) } \\
\text { that she } \\
\text { could get to } \\
\text { a health } \\
\text { facility for } \\
\text { ANC4+ [or } \\
\text { delivery] }\end{array}$} & Confident & 0.15 & 0.11 & 0.20 & $\begin{array}{l}< \\
0.001\end{array}$ & 0.15 & 0.11 & 0.18 & $\begin{array}{l}<.001 \\
0.00\end{array}$ \\
\hline & Uncertain & - & - & - & - & - & - & - & - \\
\hline & $\begin{array}{l}\text { Don't } \\
\text { know }\end{array}$ & -0.01 & -0.11 & 0.11 & 0.986 & 0.08 & -0.03 & 0.20 & 0.165 \\
\hline \multirow{2}{*}{$\begin{array}{l}\text { Besides } \\
\text { yourself, } \\
\text { who else } \\
\text { Influences } \\
\text { your } \\
\text { decision } \\
\text { about going } \\
\text { (2) Aelfetence }\end{array}$} & Partner & 0.04 & 0.00 & 0.07 & 0.029 & 0.07 & 0.04 & 0.11 & $\begin{array}{l}< \\
0.001\end{array}$ \\
\hline & $\begin{array}{l}\text { Other } \\
\text { response }\end{array}$ & - & - & - & - & - & - & - & - \\
\hline
\end{tabular}


lor

delivery]?
ANC4+

Facility-based delivery

\begin{tabular}{|c|c|c|c|c|c|c|c|c|}
\hline $\begin{array}{l}\text { Mother-in- } \\
\text { law }\end{array}$ & 0.00 & -0.09 & 0.08 & 0.915 & 0.06 & -0.02 & 0.14 & 0.154 \\
\hline $\begin{array}{l}\text { Other } \\
\text { response }\end{array}$ & - & - & - & - & - & - & - & - \\
\hline $\begin{array}{l}\text { Health } \\
\text { provider }\end{array}$ & 0.03 & -0.05 & 0.11 & 0.473 & 0.09 & 0.01 & 0.17 & 0.031 \\
\hline $\begin{array}{l}\text { Other } \\
\text { response }\end{array}$ & - & - & - & - & - & - & - & - \\
\hline
\end{tabular}

\section{Sociodemographic variables}

Household Lowest

\section{wealth}

\begin{tabular}{lcccccccc}
\hline Second & 0.01 & -0.04 & 0.05 & 0.726 & 0.00 & -0.04 & 0.05 & 0.975 \\
\hline Middle & 0.00 & -0.05 & 0.04 & 0.875 & -0.02 & -0.06 & 0.03 & 0.446 \\
\hline Fourth & -0.01 & -0.06 & 0.04 & 0.722 & -0.01 & -0.06 & 0.03 & 0.576 \\
\hline Highest & 0.06 & 0.01 & 0.12 & 0.020 & 0.05 & 0.00 & 0.11 & 0.042 \\
\hline
\end{tabular}

\begin{tabular}{|c|c|c|c|c|c|c|c|c|c|}
\hline \multirow[t]{2}{*}{$\begin{array}{l}\text { Maternal } \\
\text { education }\end{array}$} & $\begin{array}{l}\text { None or } \\
\text { informal } \\
\text { education }\end{array}$ & - & - & - & - & - & - & - & - \\
\hline & $\begin{array}{l}\text { Any } \\
\text { formal } \\
\text { education }\end{array}$ & 0.05 & 0.02 & 0.09 & 0.003 & 0.04 & 0.00 & 0.07 & 0.027 \\
\hline
\end{tabular}

\begin{tabular}{|c|c|c|c|c|c|c|c|c|c|}
\hline \multirow[t]{3}{*}{$\begin{array}{l}\text { Maternal } \\
\text { age }\end{array}$} & $\begin{array}{l}15- \\
24 \text { years }\end{array}$ & - & - & - & - & - & - & - & - \\
\hline & $\begin{array}{l}25- \\
34 \text { years }\end{array}$ & 0.01 & -0.02 & 0.03 & 0.505 & -0.02 & -0.05 & 0.00 & 0.032 \\
\hline & $\begin{array}{l}35- \\
49 \text { years }\end{array}$ & 0.02 & -0.01 & 0.06 & 0.195 & -0.02 & -0.06 & 0.01 & 0.201 \\
\hline \multirow[t]{2}{*}{$\begin{array}{l}\text { Maternal } \\
\text { occupation }\end{array}$} & $\begin{array}{l}\text { Work } \\
\text { outside } \\
\text { home or } \\
\text { student }\end{array}$ & - & - & - & - & - & - & - & - \\
\hline & $\begin{array}{l}\text { No work } \\
\text { outside } \\
\text { home }\end{array}$ & -0.06 & -0.09 & -0.02 & 0.001 & 0.02 & -0.01 & 0.06 & 0.102 \\
\hline $\begin{array}{l}\text { Spousal } \\
\text { occupation }\end{array}$ & $\begin{array}{l}\text { Work } \\
\text { outside } \\
\text { home or } \\
\text { student }\end{array}$ & - & - & - & - & - & - & - & - \\
\hline
\end{tabular}

(-) Reference category 


\begin{tabular}{|c|c|c|c|c|c|c|c|c|c|}
\hline & \multicolumn{3}{|c|}{ ANC4+ } & \multicolumn{6}{|c|}{ Facility-based delivery } \\
\hline & $\begin{array}{l}\text { No work } \\
\text { outside } \\
\text { home }\end{array}$ & 0.00 & -0.06 & 0.06 & 0.938 & 0.00 & -0.05 & 0.06 & 0.942 \\
\hline \multirow[t]{2}{*}{$\begin{array}{l}\text { Spousal } \\
\text { education }\end{array}$} & $\begin{array}{l}\text { None or } \\
\text { informal } \\
\text { education }\end{array}$ & - & - & - & - & - & - & - & - \\
\hline & $\begin{array}{l}\text { Any } \\
\text { formal } \\
\text { education }\end{array}$ & 0.03 & 0.00 & 0.06 & 0.037 & 0.04 & 0.01 & 0.07 & 0.011 \\
\hline
\end{tabular}

\section{Ideational associations with facility-based delivery}

The psychosocial factors significantly associated with facility-based delivery were ANC knowledge, beliefs about health services quality, self-efficacy as well as spousal and health worker influence on decisionmaking (Table 4, Fig. 2). After adjustment for other variables, the probability of giving birth in a facility increased 6 percentage points ( $95 \% \mathrm{Cl}: 0.03-0.09, \mathrm{p}<0.001)$ if the respondent knew that women should receive four or more check-ups during pregnancy compared to those who were not aware (knowledge); increased 12 points ( $95 \% \mathrm{Cl}: 0.09-0.15, \mathrm{p}<0.001)$ if she believed that the health facility is the best place to deliver a baby (beliefs); decreased 4 points $(95 \% \mathrm{Cl}: 0.01-0.07, \mathrm{p}=0.016)$ if she believed it's better to use a traditional provider than a health facility for ANC (beliefs); increased 15 points (95\% Cl: $0.11-0.18, p<$ 0.001 ) if she felt confident that she could get to a health facility for delivery (self-efficacy); increased 7 points (95\% Cl: $0.04-0.11, \mathrm{p}<0.001$ ) if she reported that her spouse/partner supported her decision to give birth in a facility (social influence); increased 9 points ( $95 \% \mathrm{Cl}: 0.01-0.17, \mathrm{p}=0.031$ ) if she reported that a health provider supported her decision to give birth in a facility (social influence). The following sociodemographic variables were also significantly associated with facility-based delivery in the adjusted analysis: household wealth, maternal age, maternal education, and spousal education.

\section{Discussion}

Across the study area, there were low levels of ANC4 + attendance and facility-based delivery among women 15-49 years during her last pregnancy in the past two years. Current findings suggest that cognitive, emotional and social psychosocial factors played an important role in women's pregnancy and childbirth decisions. Specifically, we found that ANC knowledge and beliefs, self-efficacy in accessing services, spousal support in pregnancy and childbirth decisions, and positive perceptions of maternal health services quality are among the most important ideations for SBC programs to target in northwestern Nigeria in order to increase ANC4 + attendance and facility-based delivery.

Within the cognitive domain, knowledge of ANC timing and benefits was significantly associated with attending ANC4 + times and giving birth in a facility. This includes knowing the recommended number of ANC check-ups and when to initiate the first ANC visit, as well as reporting at least one ANC benefit to 
herself. Indeed, it is well-recognized that raising knowledge about health behaviors, including pregnancy and childbirth, is an essential first step for health promotion activities.(16) Current results suggest that SBC programs in northwestern Nigeria may need to focus on increasing basic ANC knowledge as well as promoting the benefits of ANC and facility-based delivery among women in order to increase their use of these services.

At the same time, SBC programs must also go beyond knowledge to address cognitive beliefs that were shown to further impede progress. In our study, women who believed that pregnant women attending ANC4 + times had safer pregnancies were 1.3-times more likely to attend ANC4 + times than those who did not, while women who thought ANC was only for sick pregnant women had 17\% lower likelihood of ANC4 + attendance. The perception that ANC is only necessary if illness complications arise aligns with previous research suggesting that women often perceive pregnancy as a normal condition and place low value on antenatal care when feeling well. $(27,28)$ It also dovetails with the most common reason cited by women in our study for non-use of ANC services, notably a lack of perceived need. SBC programs may therefore need to reinforce the importance of ANC to women and children even during healthy pregnancies without complications. Women's beliefs about local health services quality were also associated with ANC4 + attendance and facility-based delivery, which underscores long-standing evidence that quality of health services is a main determinant of its use. $(29,30)$

Within the emotional dimension, self-efficacy was a significant ideational domain associated with use ANC4 + attendance and facility-based delivery. We found that women who felt confident that they could get to a facility for ANC or delivery were 2.5- and 3.4-times more likely to attend ANC4 + times or give birth in a facility than women who lacked such confidence. Indeed, inability to access facilities due to transport or other issues is a common barrier to service uptake.(31) Yet, confidence in one's own ability to undertake the behavior is an important behavioral influence itself, and further research should explore the complex reasons women may feel uncertain around accessing care for pregnancy and childbirth.(21)

Within the social dimension, supportive spousal influence on decision-making was significantly associated with both ANC4 + attendance and facility-based delivery, while health provider influence was only associated with facility-based delivery. The important role of husbands in household decision-making in northwestern Nigeria is well-established,(32) and this power dynamic is amplified by early marriage practices, polygyny, and low female literacy and formal schooling.(33) Some research suggests that men in this area may consider pregnancy and childbirth a woman's domain, and may not engage in decisionmaking even if the wife is not empowered to make such decisions alone.(32) Nevertheless, shared healthcare decision-making and male engagement in pregnancy and childbirth decisions has been shown to improve pregnancy outcomes. $(34,35)$ Our findings further underscore the important role of spousal support for uptake of maternal health services in this area. These results suggest that SBC programs should place a priority on male engagement in order to positively improve maternal health outcomes. We also found that health worker support had a positive influence on women's decision to give birth in a facility. Since providers are a conduit for positive health messaging through interpersonal communication with clients, encouraging providers to counsel women on the value of facility-based delivery during ANC visits may be an important channel for promoting behavior change. 
The results presented in this paper should be viewed in light of some methodological limitations. First, associations found in cross-sectional studies do not imply causation, and importantly, there is potential for reverse causation such that performing a behaviour (e.g. giving birth in a facility) may create or reinforce an ideation (e.g. confidence in accessing a facility for delivery). Second, observational studies are prone to residual confounding from unmeasured variables, such as ideations that were not measured or facility-level variables that were not collected as part of this household-based survey. Third, self-reported attitudes may be affected by social desirability concerns or desires to please the interviewer, which could bias responses towards more agreeable ones. Respondents' attitudes may also vary depending on the respondent's disposition at the time of the interview. Fourth, psychosocial metrics may not adequately capture the broader ideational domain, which could lead to non-significant findings for an ideation despite its potential importance for pregnancy and childbirth decisions.

\section{Conclusions}

We found that improving pregnancy and childbirth practices in northwestern Nigeria will require programs to consider addressing a wide range of psychosocial factors including raising knowledge and dispelling myths, building women's confidence to access services, engaging spousal support in decision-making, and improving perceived (and actual) maternal health services quality. To date, there has been limited research to measure and quantitatively examine the relationships between psychosocial influences and uptake of essential maternal health services in Nigeria or other LMICs. Ideational metrics provide important insights for programs that aim to change health behaviors, including pregnancy and childbirth, and should be explored for other health areas as well.

\section{Abbreviations}

ANC Antenatal care

ANC4+ Antenatal care attendance at least four time during pregnancy

DHS Demographic and Health Survey

LMIC Low- and middle-income country

SBC Social and behavior change

\section{Declarations}

\section{Ethics approval and consent to participate}

Ethical approval for this study was obtained from the National Health Research Ethics Committee in Nigeria [NHREC/01/01/2007-02/09/2019] and the Tulane University Institutional Review Board in Louisiana, USA [2019-1047]. Written informed consent to participate in the survey was obtained from all willing participants for the household and female questionnaires. Each participant signed or marked her thumbprint on the 
consent form to signify willingness to participate. All survey participants were married women and any respondent under 16 years was considered an emancipated minor not requiring parental consent.

\section{Consent to publish}

Not applicable.

\section{Availability of data and material}

The datasets used and/or analyzed during the current study are available from the corresponding author on reasonable request.

\section{Competing interests}

The authors declare that they have no competing interests.

\section{Funding}

The research was funded by the United States Agency for International Development (USAID) through the Breakthrough RESEARCH cooperative agreement [AID-OAA-A-17-00018]. The funders had no role in the study design, data collection and analysis, data interpretation, manuscript preparation or the decision to submit for publication.

\section{Authors' contributions}

PLH and PCH designed and conceptualized the study. PLH EWJ developed the study questionnaire, supervised fieldwork and data collection. EWJ UA PLH compiled, prepared, and analyzed data. EWJ UA DA MO SA-A PCH PLH contributed to interpretation of findings. EWJ wrote the first draft of the paper. EWJ UA DA MO SA-A PCH PLH reviewed, revised and contributed writing to the paper. All authors read and approved the final manuscript.

\section{Acknowledgements}

Not applicable.

\section{References}

1. World Health Organization. Trends in maternal mortality: 1990 to 2015. Geneva: WHO; 2015.

2. Federal Government of Nigeria and The DHS Program. Nigeria Demographic and Health Survey 2018. Abuja, Nigeria and Maryland, USA: Federal Government of Nigeria; 2019.

3. Souza JP. The Nigeria Near-Miss and Maternal Death Survey: collaborative research generating information for action. Bjog. 2019;126 Suppl 3:5-6.

4. Federal Government of Nigeria. National Strategic Health Development Plan II (NSHD) 2018-2022. Abuja: Federal Government of Nigeria; 2019. 
5. United Nations. Transforming our world: the 2030 agenda for sustainable development - resolution adopted by the General Assembly on 25 September 2015 A/RES70/1.2015. New York: United Nations; 2015.

6. World Health Organization. WHO recommendations on antenatal care for a positive pregnancy experience. Geneva: WHO; 2016.

7. Sageer R, Kongnyuy E, Adebimpe WO, Omosehin O, Ogunsola EA, Sanni B. Causes and contributory factors of maternal mortality: evidence from maternal and perinatal death surveillance and response in Ogun state, Southwest Nigeria. BMC Pregnancy Childbirth. 2019;19(1):63.

8. Federal Government of Nigeria. Antenatal care: an orientation package for health providers. Abuja: Nigeria Federal Ministry of Health; 2017.

9. Pedersen J, Liu J. Child mortality estimation: appropriate time periods for child mortality estimates from full birth histories. PLoS medicine. 2012;9(8):e1001289.

10. Meh C, Thind A, Ryan B, Terry A. Levels and determinants of maternal mortality in northern and southern Nigeria. BMC Pregnancy and Childbirth. 2019;19(1):417.

11. Adamu YM, Salihu HM. Barriers to the use of antenatal and obstetric care services in rural Kano, Nigeria. J Obstet Gynaecol. 2002;22(6):600-3.

12. Doctor HV, Findley SE, Ager A, Cometto G, Afenyadu GY, Adamu F, et al. Using community-based research to shape the design and delivery of maternal health services in Northern Nigeria. Reprod Health Matters. 2012;20(39):104-12.

13. Fagbamigbe AF, Idemudia ES. Barriers to antenatal care use in Nigeria: evidences from non-users and implications for maternal health programming. BMC Pregnancy Childbirth. 2015;15:95.

14. Ishola G, Fayehun F, Isiugo-Abanihe U, Segun T, Yusuf S, Orji B, et al. Effect of Volunteer Household Counseling in Improving Knowledge of Birth Preparedness and Complication Readiness of Pregnant Women in Northwest Nigeria. Afr J Reprod Health. 2017;21(1):39-48.

15. Fetohy EM. Impact of a simple health education program about antenatal care on knowledge, attitudes, subjective norms and intention of pregnant women. J Egypt Public Health Assoc. 2004;79(34):283-310.

16. Kincaid D, Delate R, Storey J, Figeroa M. Closing the gaps in practice and in theory: evaluation of the scrutinize HIV campaign in South Africa. Rice R, Atkin C, editors. Newbury Park, CA: Sage; 2013.

17. Breakthrough ACTION and RESEARCH. Breakthrough Action and Research for social and behavior change. Baltimore, Maryland: JHUCCP; 2020.

18. Johansson EW, Hutchinson PL, Omoluabi E, Akinyemi A, Smith A, Welty JC, et al. Behavioral sentinel surveillance survey in Nigeria: baseline technical report. Washington DC: Population Council; 2020.

19. National Bureau of Statistics. Demographics Statistics Bulletin. Abuja: National Bureau of Statistics; 2017.

20. Storey JD, Babalola SO, Ricotta EE, Fox KA, Toso M, Lewicky N, et al. Associations between ideational variables and bed net use in Madagascar, Mali, and Nigeria. BMC public health. 2018;18(1):484. 
21. Babalola S, John N, Ajao B, Speizer IS. Ideation and intention to use contraceptives in Kenya and Nigeria. Demogr Res. 2015;33:211-38.

22. Larson HJ, Jarrett C, Schulz WS, Chaudhuri M, Zhou Y, Dube E, et al. Measuring vaccine hesitancy: The development of a survey tool. Vaccine. 2015;33(34):4165-75.

23. Amoakoh-Coleman M, Ansah EK, Agyepong IA, Grobbee DE, Kayode GA, Klipstein-Grobusch K. Predictors of skilled attendance at delivery among antenatal clinic attendants in Ghana: a crosssectional study of population data. BMJ Open. 2015;5(5):e007810.

24. Okedo-Alex IN, Akamike IC, Ezeanosike OB, Uneke CJ. Determinants of antenatal care utilisation in subSaharan Africa: a systematic review. BMJ Open. 2019;9(10):e031890.

25. Aliyu AA, Dahiru T. Predictors of delayed Antenatal Care (ANC) visits in Nigeria: secondary analysis of 2013 Nigeria Demographic and Health Survey (NDHS). Pan Afr Med J. 2017;26:124-.

26. Filmer D, Pritchett L. Estimating wealth effects without expenditure data- or tears : an application to educational enrollments in states of India. Washington, DC: World Bank; 1998. 38 p. p.

27. Warri D, George A. Perceptions of pregnant women of reasons for late initiation of antenatal care: a qualitative interview study. BMC Pregnancy and Childbirth. 2020;20(1):70.

28. Finlayson K, Downe S. Why do women not use antenatal services in low- and middle-income countries? A meta-synthesis of qualitative studies. PLoS medicine. 2013;10(1):e1001373-e.

29. Kruk ME, Hermosilla S, Larson E, Mbaruku GM. Bypassing primary care clinics for childbirth: a crosssectional study in the Pwani region, United Republic of Tanzania. Bulletin of the World Health Organization. 2014;92(4):246-53.

30. Larson E, Gage AD, Mbaruku GM, Mbatia R, Haneuse S, Kruk ME. Effect of a maternal and newborn health system quality improvement project on the use of facilities for childbirth: a cluster-randomised study in rural Tanzania. Tropical medicine \& international health : TM \& IH. 2019;24(5):636-46.

31. Bohren MA, Hunter EC, Munthe-Kaas HM, Souza JP, Vogel JP, Gülmezoglu AM. Facilitators and barriers to facility-based delivery in low- and middle-income countries: a qualitative evidence synthesis. Reproductive health. 2014;11(1):71-.

32. Oguntunde O, Nyenwa J, Yusuf FM, Dauda DS, Salihu A, Sinai I. The experience of men who participated in interventions to improve demand for and utilization of maternal and child health services in northern Nigeria: a qualitative comparative study. Reproductive health. 2019;16(1):104-.

33. Wolf M, Abubakar A, Tsui S, Williamson N. Child Spacing Attitudes in Northern Nigeria. Washington, D.C.: Family Health International; 2008.

34. Danforth EJ, Kruk ME, Rockers PC, Mbaruku G, Galea S. Household decision-making about delivery in health facilities: evidence from Tanzania. J Health Popul Nutr. 2009;27(5):696-703.

35. Yargawa J, Leonardi-Bee J. Male involvement and maternal health outcomes: systematic review and meta-analysis. Journal of Epidemiology and Community Health. 2015;69(6):604-12.

\section{Figures}


90.0

aNC4+ a Facility-based delivery

80.0

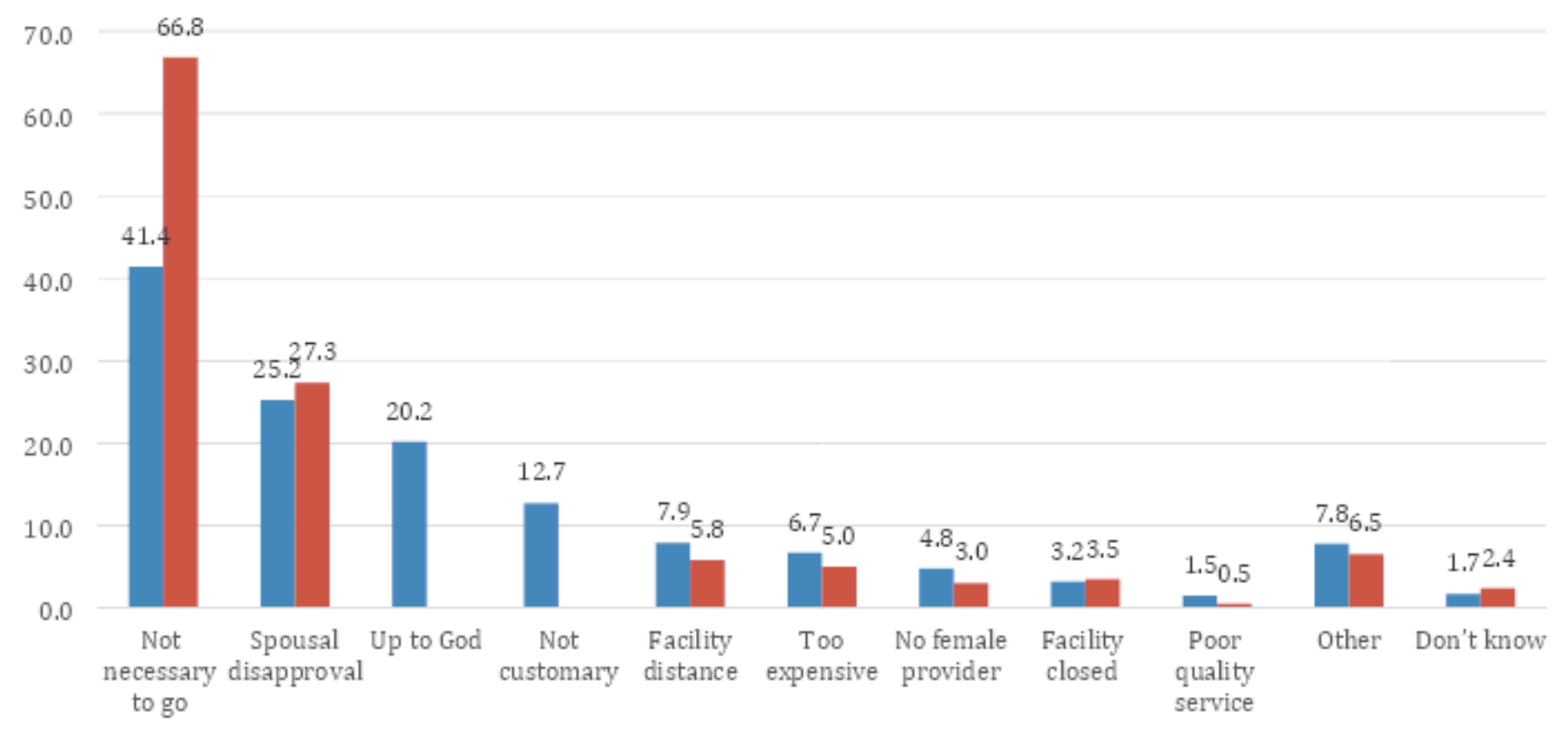

Figure 1

Reasons for non-use of antenatal care and facility-based delivery services 
90.0

aNC4+ a Facility-based delivery

80.0

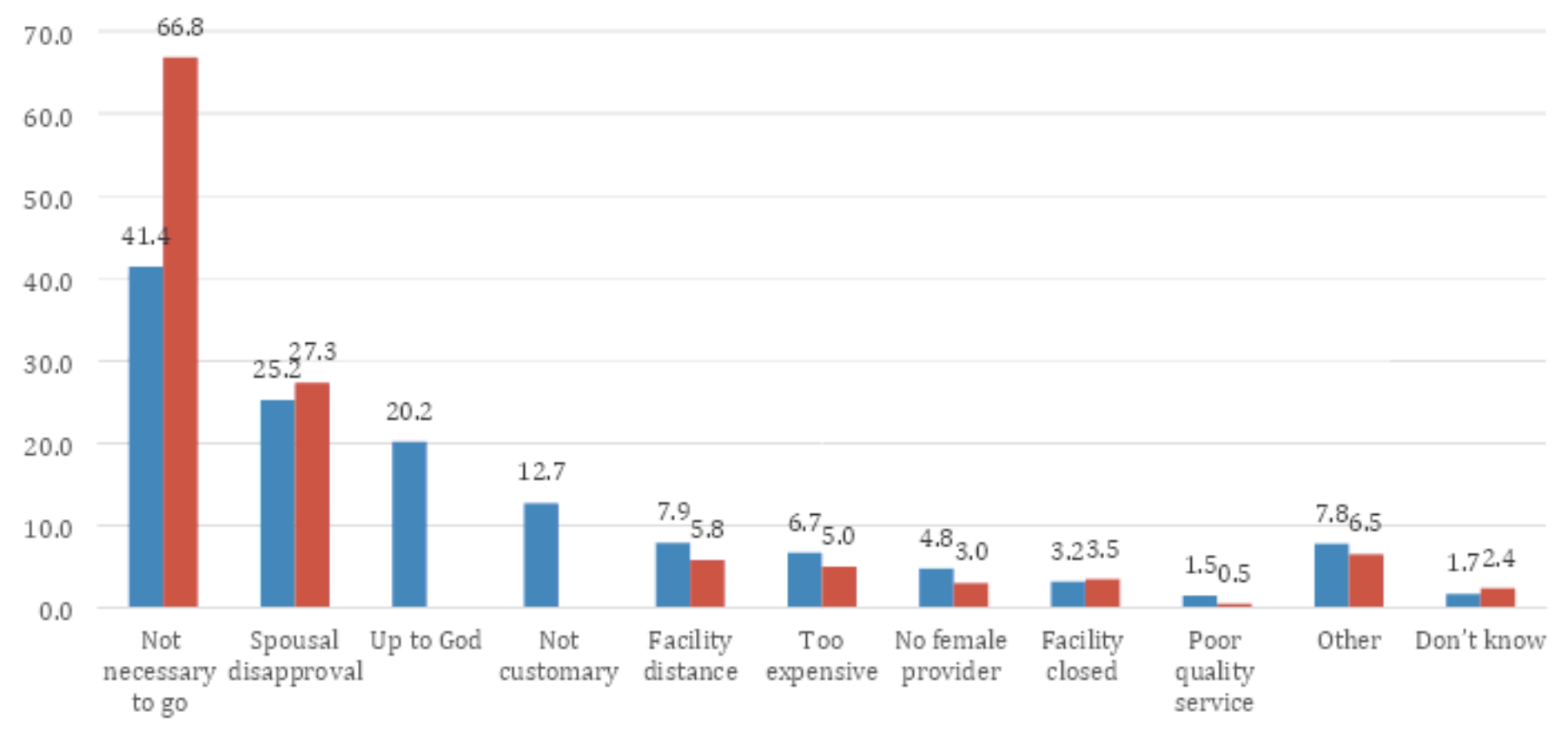

Figure 1

Reasons for non-use of antenatal care and facility-based delivery services 


\section{a) ANC4+}

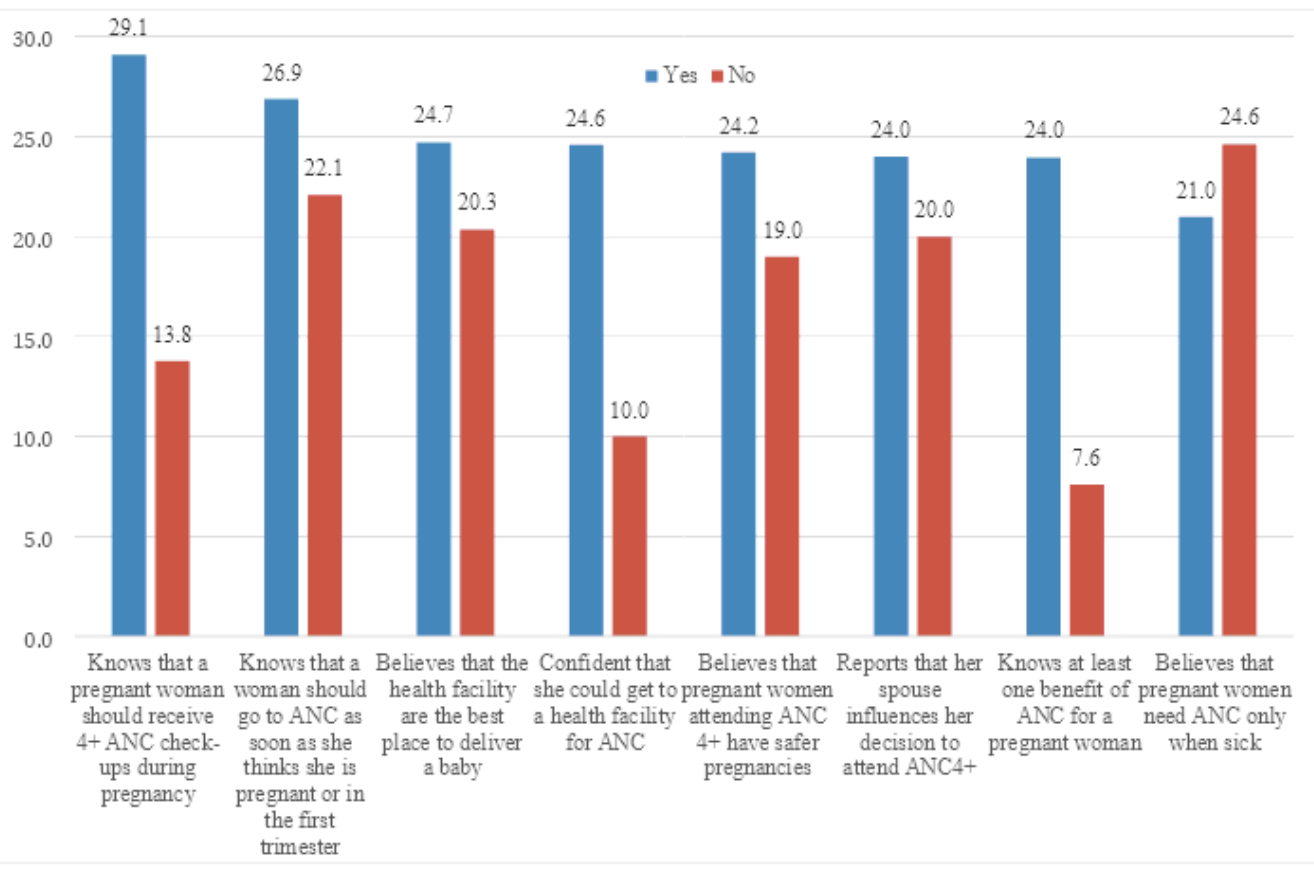

\section{b) Facility-based delivery}

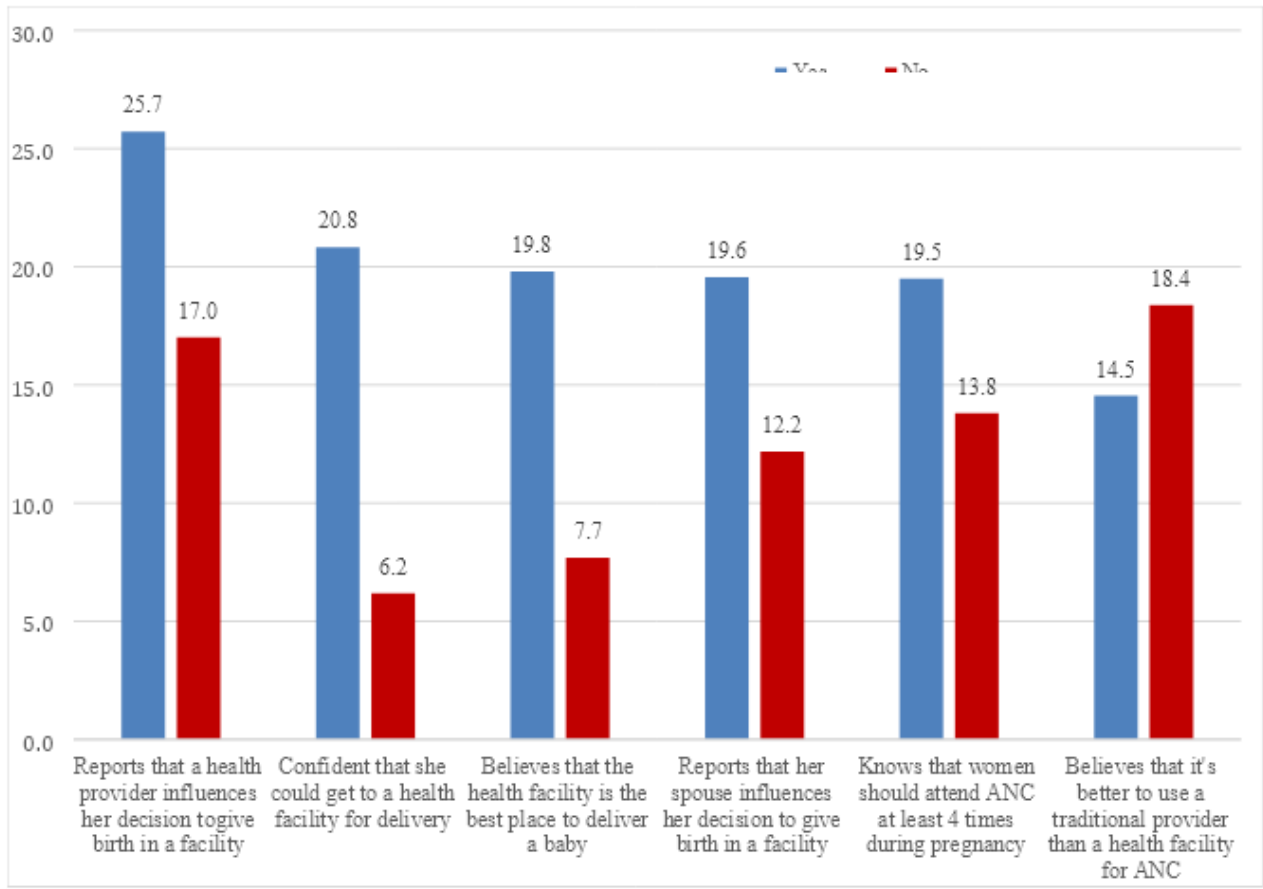

Figure 2

Predicted probabilities of ANC4+ and facility-based delivery outcomes among those with (or without) significant ideational characteristics 


\section{a) ANC4+}

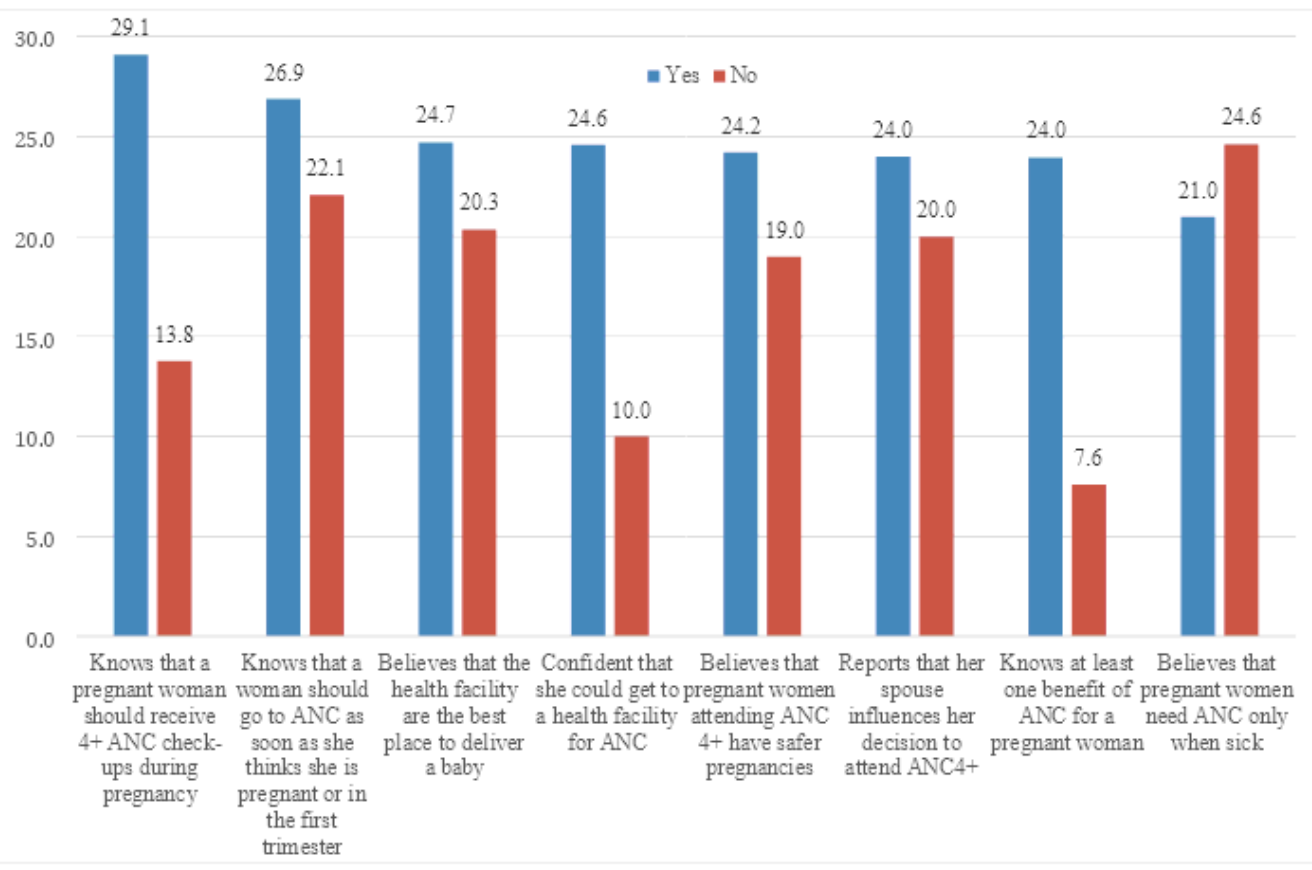

b) Facility-based delivery

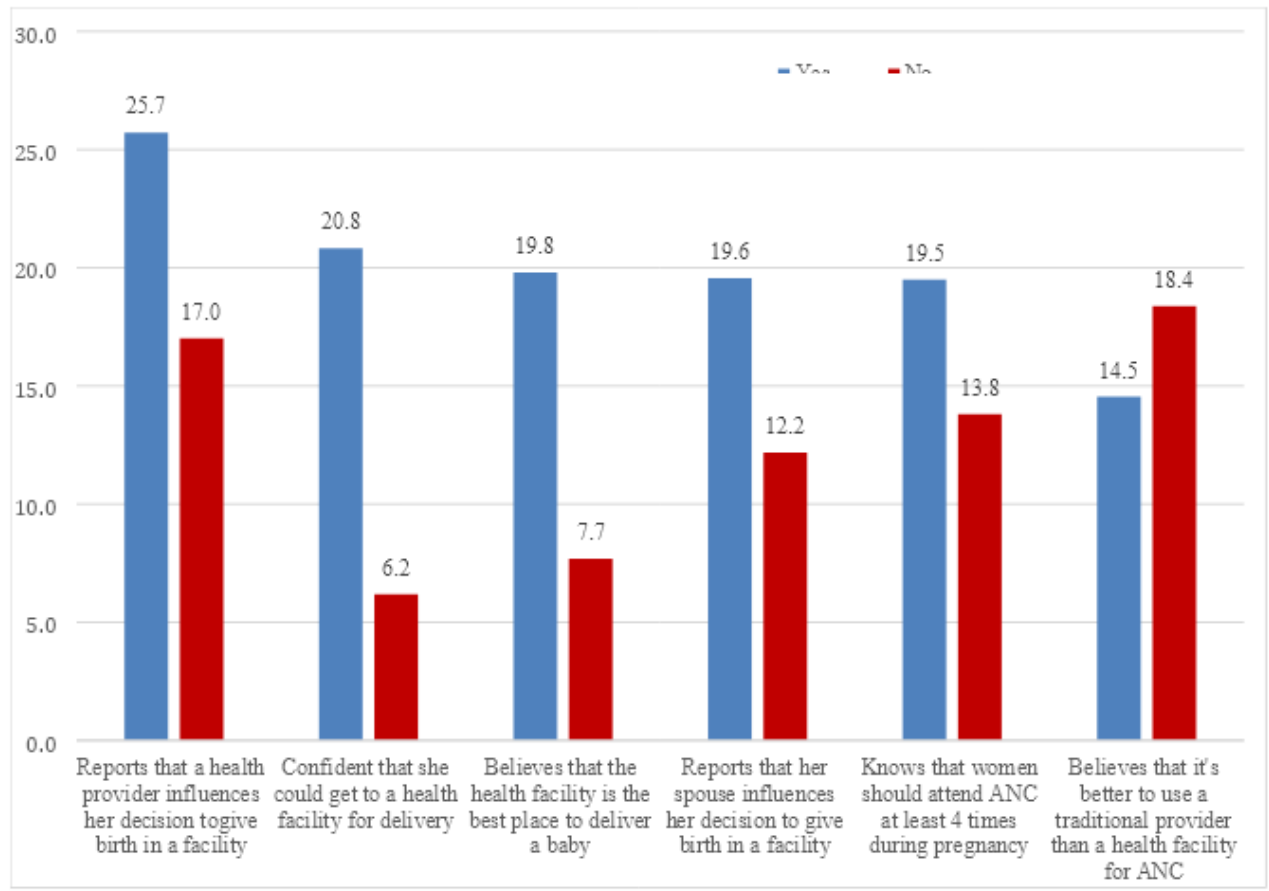

Figure 2

Predicted probabilities of ANC4+ and facility-based delivery outcomes among those with (or without) significant ideational characteristics

\section{Supplementary Files}


This is a list of supplementary files associated with this preprint. Click to download.

- 20190911BSSQuestionnaireFinalNoCategories.docx

- 20190911BSSQuestionnaireFinalNoCategories.docx 